Check for updates

Cite this: RSC Adv., 2017, 7, 33929

Received 12th May 2017

Accepted 22nd June 2017

DOI: $10.1039 / c 7 r a 05383 e$

rsc.li/rsc-advances

\section{Difunctional Cu-doped carbon dots: catalytic activity and fluorescence indication for the reduction reaction of $p$-nitrophenol $\uparrow$}

\author{
Jinyan Du, (iD * Yun Zhao, Juan Chen, Ping Zhang, Lingling Gao, Meiqin Wang, \\ Cong Cao, Wu Wen and Changqing Zhu*
}

\begin{abstract}
Herein, we report the preparation of $\mathrm{Cu}$-doped carbon dots (CDs) through a one-step hydrothermal carbonization using $\mathrm{CuCl}_{2} \cdot 2 \mathrm{H}_{2} \mathrm{O}$ and ethylenediaminetetraacetic acid disodium salt dihydrate (EDTA) as precursors. As confirmed by Fourier transform infrared (FTIR) and X-ray photoelectron spectroscopy (XPS), the $\mathrm{Cu}$ species chelates with the carbon matrix through $\mathrm{Cu}-\mathrm{N}$ complexes. Using $\mathrm{p}$-nitrophenol reduction as a model reaction, the catalytic activity and performance as a fluorescence indicator in the catalytic process are further examined. Results show that the synergistic effect, which may stem from the highly efficient catalysis activity of $\mathrm{Cu}$ and the electron-enhanced effect from graphite-like CDs, is responsible for the catalytic activity of $p$-nitrophenol hydrogenation reaction with the pseudo-first-order rate constant being $1.2 \times 10^{-2} \mathrm{~s}^{-1}$. Interestingly, the catalyzed reduction process of $p$-nitrophenol can be tracked using fluorescence spectra of Cu-doped CDs by means of the Inner Filter Effect (IFE). The unique properties of the difunctional $\mathrm{Cu}$-doped $\mathrm{CD}$ catalyst as well as the IFE sensing strategy will provide an ideal platform to monitor the catalytic processes.
\end{abstract}

\section{Introduction}

Catalytic reactions are a widespread type of chemical reaction in the natural world., ${ }^{1,2}$ Development of catalysts with excellent catalytic efficiency as well as trackable catalytic reaction dynamics is important in the research field of catalytic reactions. Within this, indication of catalytic reaction processes is of particular significance. At present, several schemes have been reported. For a system with absorbance changes, the absorption spectral changes of the reactants or products are used to monitor the catalytic process. ${ }^{3,4}$ If no absorbance change occurs in the system, the catalytic process is often tracked using spectrographic signals from additional chromogenic agents., Sometimes, the signals of chemical luminescence are used to indicate a catalytic process, i.e., for the luminol- $\mathrm{H}_{2} \mathrm{O}_{2}$ system. ${ }^{7,8}$ In fact, whether the spectrum signal changes or not, nuclear magnetic resonance (NMR) is a universal method that not only can track the catalytic reaction process, but also can capture the reaction mechanism. ${ }^{\mathbf{9 , 1 0}}$ However, it is hard to use NMR for in situ, real-time and fast tracking of the catalytic reaction process. In all, these indication means are far from meeting the

Anhui Key Laboratory of Chemo-Biosensing, Key Laboratory of Functional Molecular Solids, Ministry of Education, College of Chemistry and Materials Science, Anhui Normal University, Wuhu, 241000, PR China. E-mail: dujinyn@mail.ahnu.edu.cn; zhucq@mail.ahnu.edu.cn

$\uparrow$ Electronic supplementary information (ESI) available. See DOI: 10.1039/c7ra05383e requirements where both good catalytic performance and sensitive indicative function are needed in the catalytic reactions. Moreover, it is noted that most of the current catalytic reaction process was monitored by the signals produced from the substrates or products. Can the catalytic reaction process be indicated by the catalyst's own signals?

In recent years, due to low toxicity, good biocompatibility, excitation-dependent fluorescence feature and high resistance to photobleaching, luminescent carbon nanomaterials have been widely applied in the fields of sensing, ${ }^{11,12}$ biological images ${ }^{13,14}$ and drug release, ${ }^{15,16}$ etc. It is worth mentioning that fluorescence carbon dots (CDs) have an advantage of unique electron transfer, indicative of a great potential as catalyst. Extensive works related to the CDs as electrocatalyst/ photocatalyst/redox catalysts or mimic enzymes have been reported. ${ }^{17-20}$ For instances, Qu et al. ${ }^{17}$ have reported that their asprepared nitrogen-doped graphene quantum dots (N-GQDs) were able to be used as metal-free oxygen reduction reaction catalysts in fuel cells. Shi and coworkers ${ }^{18}$ demonstrated that the CDs have an intrinsic peroxidase-like activity to oxidation 3,3,5,5-tetramethylbenzidine (TMB) by hydrogen peroxide.

More interestingly, because of the modulation in the band structure, metal atom doping not only can improve the optical properties but also can vest novel functionalities to the CDs. For examples, hydrogen production efficiency are greatly enhanced with the doping of nickel because the absorbed UV and visible light can directly transfer its photoexcited electrons to solution. ${ }^{21}$ Doping with gadolinium was demonstrated to be used as 
a magnetic resonance imaging positive contrast agent. ${ }^{22}$ Moreover, $\mathrm{Zn}$-doped CDs can be served as a fluorescent probe for the detection of $\mathrm{H}_{2} \mathrm{O}_{2}$ and glucose based on the Fenton reaction ${ }^{23}$ while doping with both $\mathrm{Cu}$ and $\mathrm{N}$ atoms can be acted as a novel photocatalyst because of the enhanced electron-accepting and donating ability. ${ }^{24}$ Inspired by these observations, herein, we report the preparation of $\mathrm{Cu}$-doped $\mathrm{CDs}$ by a one-step hydrothermal carbonization of $\mathrm{CuCl}_{2} \cdot 2 \mathrm{H}_{2} \mathrm{O}$ and ethylenediaminetetraacetic acid disodium salt dihydrate (EDTA). The catalytic reduction of $p$-nitrophenol in the presence of $\mathrm{NaBH}_{4}$ was chosen as a model reaction to investigate the catalytic activity of as-obtained $\mathrm{Cu}$-doped CDs. On the basis of inner filter effect (IFE), the fluorescence excitation spectra of CDs were tuned to overlap with the absorption spectra of $p$-nitrophenol in the catalyzed system for investigating fluorescence indication performance to the catalytic reduction process of $p$-nitrophenol. Finally, the proposed catalytic mechanism and the rate of reaction were further investigated in detail.

\section{Experimental section}

\subsection{Chemicals}

The $p$-nitrophenol (99\%) and sodium borohydride $\left(\mathrm{NaBH}_{4}\right)$ were purchased from Alfa Aesar (Karlsruhe, Germany). Cupric chloride dihydrate $\left(\mathrm{CuCl}_{2} \cdot 2 \mathrm{H}_{2} \mathrm{O}, 99 \%\right)$, ethylenediaminetetraacetic acid disodium salt dihydrate (EDTA, 98\%) and other routine chemicals were acquired from Guoyao Chemical Reagent Company (Shanghai, China) and used as received without further purification. All solutions were prepared with ultrapure water $(18.25 \mathrm{M} \Omega \mathrm{cm})$.

\subsection{Preparation of Cu-doped CDs}

Typically, $1.0 \mathrm{~g}$ of EDTA and $0.458 \mathrm{~g}$ of $\mathrm{CuCl}_{2} \cdot 2 \mathrm{H}_{2} \mathrm{O}$ were dispersed in $25 \mathrm{~mL}$ water by sonication for $0.5 \mathrm{~h}$. Afterward the above mixture solution was transferred into a $50 \mathrm{~mL}$ Teflon-lined autoclave. Then the reaction system was sealed and heated from room temperature to $200{ }^{\circ} \mathrm{C}$ and kept at that temperature for $4 \mathrm{~h}$. After the resultant solution was cooled to room temperature naturally, the products were collected by filtering, centrifuging and the as-collected supernatant was dialyzed in the dialysis bag (molecular weight: $500 \mathrm{kDa}$ ) for $24 \mathrm{~h}$. The purified CDs were preserved at $4{ }^{\circ} \mathrm{C}$ for the following experiments.

\subsection{Preparation of bare CDs}

The preparation process of the bare CDs is the same to the $\mathrm{Cu}$ doped CDs while the only exception is that $\mathrm{CuCl}_{2} \cdot 2 \mathrm{H}_{2} \mathrm{O}$ needs not to be added into the reaction system.

\subsection{Characterization}

The morphology and microstructure were investigated by transmission electron microscopy (TEM, JEOL 2010, Japan). X-ray photoelectron spectroscopy (XPS) was measured on a thermoelectron instrument (Thermo-VG Scientific ESCALAB MK II). Elemental analysis was carried out by energy dispersive X-ray spectroscopy (EDS) conducted at the accelerating voltage of 200 $\mathrm{kV}$ on a Philips S-4800 instrument. UV-vis spectra of the samples were obtained on a Hitachi UV-2910 spectrophotometer. The fluorescence spectra were recorded with a Hitachi F-4600 fluorescence spectrophotometer. Fourier transform infrared (FTIR) spectra were carried out on a PerkinElmer PE-983 FTIR spectrophotometer.

\subsection{Catalytic assay toward $\boldsymbol{p}$-nitrophenol reduction reaction}

The reduction of $p$-nitrophenol by $\mathrm{NaBH}_{4}$ was chosen as a model reaction to investigate catalytic activity and indication performance of the prepared Cu-doped CDs. In a typical experiment, $1.0 \mathrm{~mL}$ of $p$-nitrophenol $\left(3.0 \times 10^{-4} \mathrm{~mol} \mathrm{~L}^{-1}\right)$ and $1.0 \mathrm{~mL}$ of $\mathrm{NaBH}_{4}\left(3.0 \times 10^{-2} \mathrm{~mol} \mathrm{~L}^{-1}\right)$ were introduced into a colorimetric tube and followed by adding different amount of $\mathrm{Cu}$-doped CDs, and then diluted to $5 \mathrm{~mL}$ with Tris-HCl buffer solution ( $\left.0.01 \mathrm{~mol} \mathrm{~L}^{-1}, \mathrm{pH} 4.0\right)$. The color of the solution changed gradually from yellow to transparent as the reaction proceeding. At the same time, the catalytic activity was monitored in situ on Hitachi UV-2910 spectrophotometer and Hitachi F-4600 fluorescence spectrophotometer.

\section{Results and discussion}

\subsection{Characterization of Cu-doped CDs}

The Cu-doped CDs were synthesized according to the protocol described in the experimental section. As shown in Fig. 1A, optical absorption in the UV region was observed in the UV-vis absorption spectrum of Cu-doped CDs. In the inset of Fig. 1A, the Cu-doped CDs emitted bright blue light under UV light
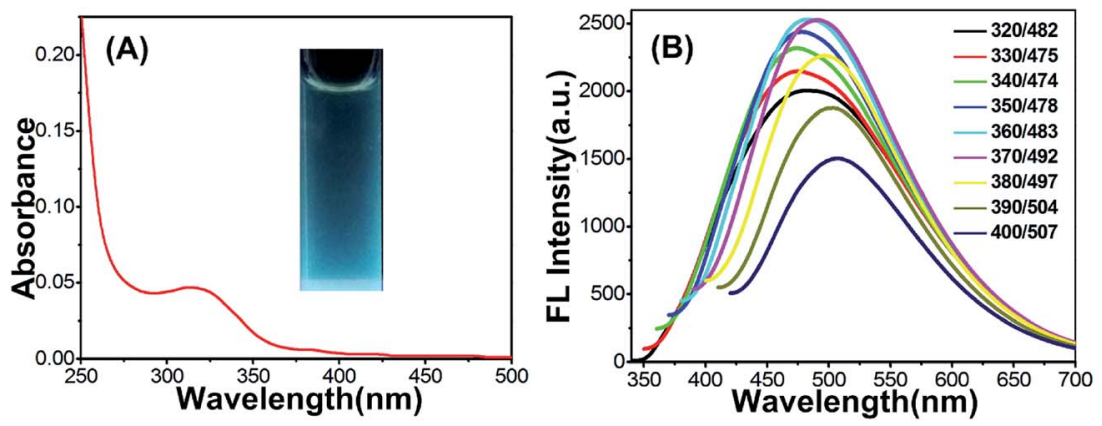

Fig. 1 (A) UV-vis absorption spectra of the Cu-doped CDs solution. Inset: the photograph of Cu-doped dispersion under UV light (365 nm). (B) Fluorescence emission spectra of the $\mathrm{Cu}$-doped dispersed in water at excitation wavelengths from $320 \mathrm{~nm}$ to $400 \mathrm{~nm}$. 

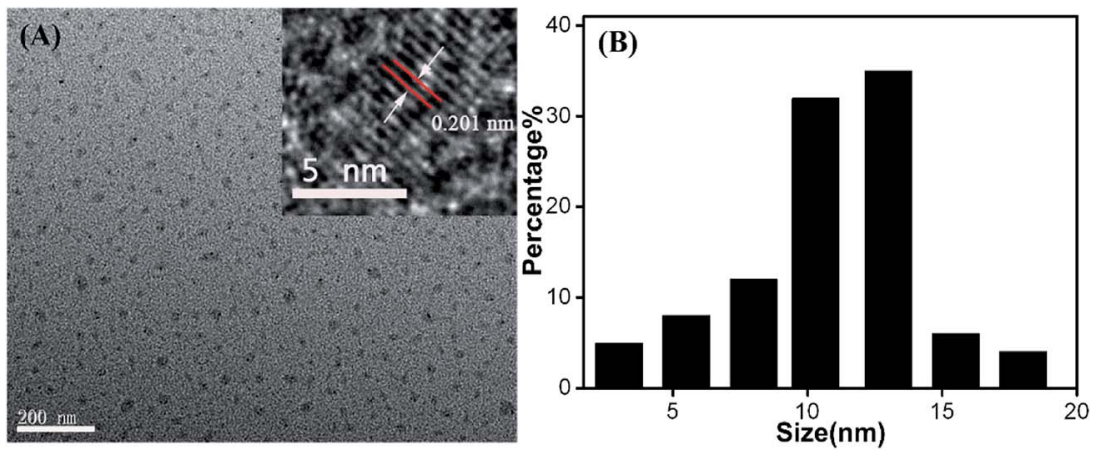

Fig. 2 (A) TEM and HRTEM (inset) images of Cu-doped CDs. (B) Size distribution of Cu-doped CDs analyzed from multiple images.
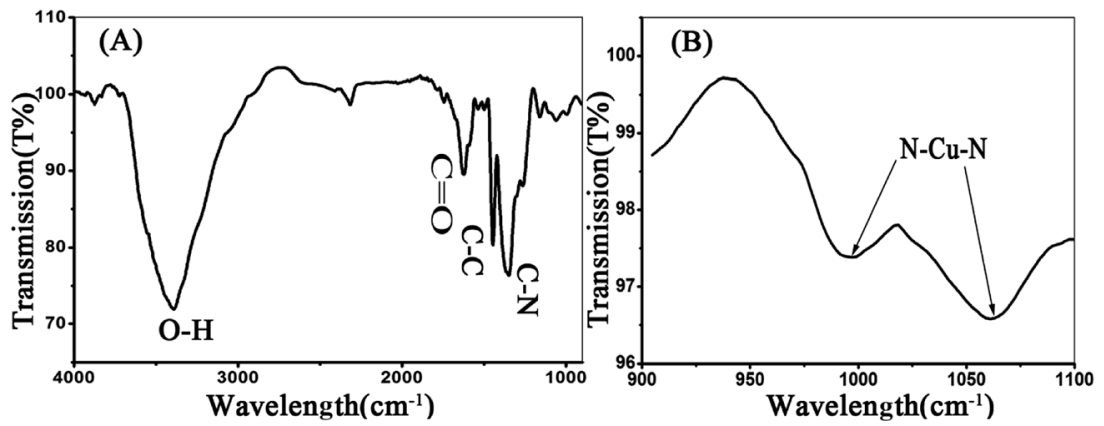

Fig. 3 (A) FTIR spectrum of Cu-doped CDs. (B) Amplified FTIR spectrum at the region of 900 to $1100 \mathrm{~cm}^{-1}$.

excitation $(365 \mathrm{~nm})$. From the fluorescent emission spectra of the Cu-doped CDs (Fig. 1B), the excitation-dependent fluorescence behavior were observed in the range from 350 to $700 \mathrm{~nm}$, which is one of most special properties of CDs (the fluorescent emission spectra of bare CDs is shown in Fig. S1† for comparison). ${ }^{25,26}$ In addition, the average fluorescence quantum yield (QY) of the CDs in aqueous solution at room temperature was measured to be $\sim 6.1 \%$ using quinine sulfate as reference ( $\mathrm{QY}$ of quinine sulfate is $54 \%$ at $370 \mathrm{~nm}$ excitation wavelength). This value is comparable with the result reported in the previous literatures for carbon dots..$^{27-29}$

The morphology of as-obtained $\mathrm{Cu}$-doped CDs was characterized by TEM and high-resolution TEM (HRTEM, Fig. 2). As can be observed in Fig. 2, an average diameter of Cu-doped CDs is about $10.5 \mathrm{~nm}$, which is larger than that of the bare CDs (an average diameter $\sim 3.5 \mathrm{~nm}$, Fig. S2 $\dagger$ ). The HRTEM image (inset in Fig. 2A) displays a lattice fringe spacing of $0.201 \mathrm{~nm}$, which may be attributed to the diffraction planes of $\mathrm{sp}^{2}$ graphitic carbon. ${ }^{30,31}$ FTIR spectrum was used to study the functional groups on the surface of CDs. As shown in Fig. 3A, the very strong absorption bands at $3386 \mathrm{~cm}^{-1}$ is ascribed to the stretching vibrations of $\mathrm{O}-\mathrm{H}$ and $\mathrm{N}-\mathrm{H}$. The high-intensity peaks at $1624 \mathrm{~cm}^{-1}, 1443$ $\mathrm{cm}^{-1}$, and $1350 \mathrm{~cm}^{-1}$ correspond to the stretching vibrations of $\mathrm{C}=\mathrm{O}, \mathrm{C}-\mathrm{C}$ and $\mathrm{C}-\mathrm{N} .^{32}$ The FTIR spectrum from 900 to $1100 \mathrm{~cm}^{-1}$ was amplified to further characterize the chemical bond between the $\mathrm{Cu}$ and the matrix, as shown in Fig. 3B. Compared with the FTIR spectrum of bare CDs as shown in Fig. S3, $\uparrow$ the new peaks at 1060 and $990 \mathrm{~cm}^{-1}$ can be assigned to $\mathrm{N}-\mathrm{Cu}-\mathrm{N}$ stretching vibrations, further confirming the formation of $\mathrm{Cu}$ coordination complexes in Cu-doped CDs. ${ }^{33}$

To confirm the composition of the Cu-doped CDs, X-ray spectroscopy (EDS) measurements were performed. EDS spectrum of $\mathrm{Cu}$-doped CDs (Fig. S4 $\dagger$ ) presents the four elements of $\mathrm{C}, \mathrm{O}, \mathrm{N}, \mathrm{Cu}$ from 0 to $1.5 \mathrm{keV}$. For a better insight, X-ray photoelectron spectroscopy (XPS) in the range of $0-980 \mathrm{eV}$ was carried out. As shown in Fig. 4, the as-prepared CDs contain the elements of $\mathrm{C}, \mathrm{O}, \mathrm{N}, \mathrm{Cu}$ with the percentage of $61.93 \%, 24.17 \%$, $11.14 \%$ and $2.76 \%$, respectively. The appearance of the $\mathrm{C} 1 \mathrm{~s}$ peak (Fig. $4 \mathrm{~A}$ ) at 284.6, 285.7 and $287.1 \mathrm{eV}$, are attributed to $\mathrm{C}-\mathrm{C} /$ $\mathrm{C}=\mathrm{C}, \mathrm{C}-\mathrm{O}$ and $\mathrm{C}=\mathrm{O} / \mathrm{C}=\mathrm{N}$, respectively. And the $\mathrm{O} 1 \mathrm{~s}$ spectrum (Fig. 4B) consisting of three peaks at 532.1, 532.6 and $533.8 \mathrm{eV}$, can be ascribed to $\mathrm{C}-\mathrm{O}, \mathrm{C}=\mathrm{O} / \mathrm{N}=\mathrm{O}$ and $\mathrm{O}-\mathrm{C}=\mathrm{O} \cdot{ }^{34}$ In addition, the high-resolution $\mathrm{N}$ 1s spectrum (Fig. 4C) was mainly divided into three peaks at 398.2, 399.5 and $401.6 \mathrm{eV}$, which may be assigned to pyridinic, pyrrolic and quaternary-like environments. Compared with the XPS of bare CDs (Fig. S5 $\dagger$ ), the detailed $\mathrm{Cu} 2$ p spectrum (Fig. 4D) shows the presence of $\mathrm{Cu}$ in $\mathrm{Cu}$-doped CDs $(932.5,952.5 \mathrm{eV})$ with corresponding to the spinorbit splitting of $\mathrm{Cu} 2 \mathrm{p}_{3 / 2}$ and $\mathrm{Cu} 2 \mathrm{p}_{1 / 2}$, respectively, indicating the existence of $\mathrm{Cu}^{\mathrm{II}}$ in as-prepared $\mathrm{Cu}$-doped CDs. ${ }^{35,36}$

\subsection{Catalytic activity and indication process for catalytic reduction of $p$-nitrophenol}

The catalytic reduction of $p$-nitrophenol to $p$-aminophenol in the presence of $\mathrm{NaBH}_{4}$ was chosen as a model reaction to investigate bifunctionality with catalytic activity and indication 
performance of the as-obtained Cu-doped CDs. The initial concentrations of $p$-nitrophenol and $\mathrm{NaBH}_{4}$ were kept at $1.0 \times$ $10^{-4}$ and $1.0 \times 10^{-2} \mathrm{~mol} \mathrm{~L}^{-1}$, respectively, in all the reactions. $p$ Nitrophenol disappearance and $p$-aminophenol generation were monitored by UV-vis absorption spectra. Meanwhile, the fluorescence spectra of the Cu-doped CDs were in situ tracked during the reaction process. As shown in Fig. S6, $\uparrow$ the original absorption peak of $p$-nitrophenol is centered at $317 \mathrm{~nm}$ and shifts to $400 \mathrm{~nm}$ after the addition of freshly prepared $\mathrm{NaBH}_{4}$ solution, indicating that the sodium $p$-nitrophenolate ions are formed. ${ }^{37}$ The peak at $400 \mathrm{~nm}$ is constant and no new peak at $300 \mathrm{~nm}$ is appeared in a period time, which indicates that the reduction reaction does not take place in the absence of catalyst. However, when a small amount of as-synthesized Cu-doped CDs were added, 30 seconds later, the reaction system becomes stable. Afterward the absorption peak at $400 \mathrm{~nm}$ was gradually decreased with the concomitant intensity increase of new peak at ca. $295 \mathrm{~nm}$ (Fig. 5A), and the yellow color of the reaction mixture was faded within 5 min (inset of Fig. 5A), demonstrating the occurrence of the reduction reaction and the formation of $p$-aminophenol. ${ }^{38-40}$ But the absorption peak at $400 \mathrm{~nm}$ was unchanged by using the bare CDs as the catalyst (Fig. S7A $\dagger$ ), suggesting that the reduction could not be occurred in the absence of $\mathrm{Cu}$.

The reduction of $p$-nitrophenol to $p$-aminophenol can be treated as a pseudo-first-order reaction because the concentration of $\mathrm{NaBH}_{4}$ is higher than that of $p$-nitrophenol which can be considered as a constant during the reaction period. ${ }^{41}$ The values of the rate constants of the above catalytic reactions were calculated from the plot of $\ln \left(c_{t} / c_{0}\right)$ versus time $t$. Here, $c_{t}$ presents the concentration of $p$-nitrophenol at time $t$, and $c_{0}$ is the initial concentration of $p$-nitrophenol. The $c_{t} / c_{0}$ is measured from the relative intensity of absorbance $\left(A_{t} / A_{0}\right)$ at $400 \mathrm{~nm}$. The relationship of $\ln \left(c_{t} / c_{0}\right)$ versus time $(t)$ was presented in Fig. $5 \mathrm{~B}$. The rate constant for $p$-nitrophenol reduction is calculated to be $1.23 \times$ $10^{-2} \mathrm{~s}^{-1}$. Although Cu-doped CDs exhibit a high catalytic activity
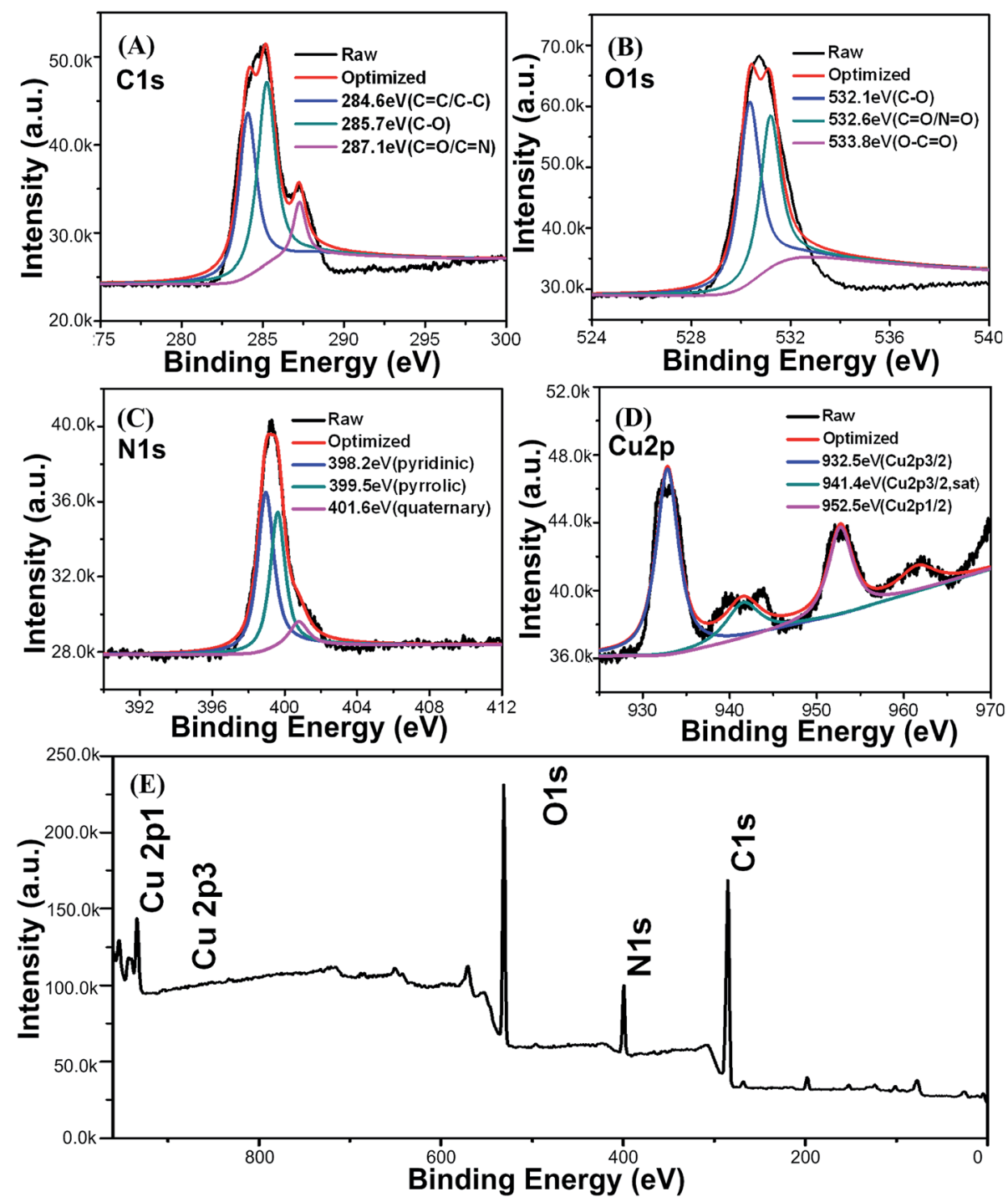

Fig. 4 XPS high-resolution survey of C 1s (A), O 1s (B), N 1s (C), Cu 2p (D) and entirety (E) of the Cu-doped CDs. 

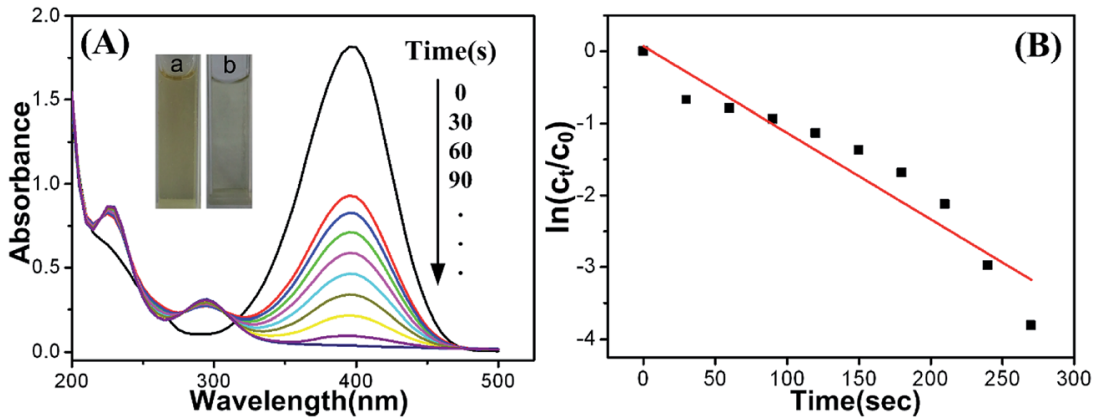

Fig. 5 (A) Time-dependent UV-vis spectral changes in p-nitrophenol solution catalyzed by $\mathrm{Cu}$-doped $\mathrm{CDs}_{\text {in }}$ the presence of $\mathrm{NaBH}_{4}$. Inset: the photograph of mixture containing $p$-nitrophenol and $\mathrm{NaBH}_{4}$ before (a) and after (b) the addition of $\mathrm{Cu}$-doped CDs under natural light. (B) Plots of $\ln \left(c_{t} / c_{0}\right)$ versus time for the catalytic reduction of $p$-nitrophenol at room temperature.

for the reduction reaction of $p$-nitrophenol to $p$-aminophenol, the bare CDs was found to be inactive (Fig. S7B†). The reason may be attributed to their heterostructures and electronic junction effect, ${ }^{42}$ which indicates that the element of Cu play a vital role in the reduction of $p$-nitrophenol. Scheme 1 shows a proposed catalytic mechanism for $p$-nitrophenol reduction by $\mathrm{Cu}$-doped CDs in the presence of $\mathrm{NaBH}_{4}$. Each $\mathrm{Cu}$ atom includes unpaired electrons, these unpaired electrons could interact with the adsorbed $p$-nitrophenol by forming a chemical adsorption bond upon adsorption..$^{43}$ During the hydrogenation reduction of $p$-nitrophenol, the generated $p$-nitrophenolate ions and $\mathrm{BH}_{4}{ }^{-}$ were adsorbed on the electronic hole in the surface of the $\mathrm{Cu}$ via chemical adsorption and immediately followed by the formation of $p$-aminophenol through hydrogenation reduction of $p$-nitrophenol on the surface of $\mathrm{Cu}$. In order to confirm that the CDs can absorb the electron negative $p$-nitrophenol, $p$-nitrophenolate ions and $\mathrm{BH}_{4}{ }^{-}$, zeta potential of Cu-doped-CDs in Tris-HCl buffer solution (0.01 $\mathrm{mol} \mathrm{L}^{-1}$, pH 4.0) was measured, which is $\sim 16.1 \mathrm{mV}$. Moreover, with the reduction of $p$-nitrophenol proceeding, zeta potential gradually becomes negative. In addition, CDs with graphite-like structure should be beneficial for enhancing conductivity in the electron-transfer process. ${ }^{32,44}$ Thus, the synergistic effect of $\mathrm{Cu} /$ graphitic carbon nanocatalysts finally exhibits highly catalytic performance for the hydrogenation reduction of $p$-nitrophenol to $p$-aminophenol with the rate constant of $1.23 \times 10^{-2} \mathrm{~s}^{-1}$. In Table 1 , we compare our rate constant result to those reported in the literatures on the $\mathrm{Cu}$ contained catalyst. The reasons why $\mathrm{Cu}$ is frequently chosen as

Table 1 Recent studies on the reduction of 4-NP over Cu-contained catalysts

\begin{tabular}{lll}
\hline Catalysts & Rate constant $k\left(\mathrm{~s}^{-1}\right)$ & Reference \\
\hline Cu NPs & $3.7 \times 10^{-4}$ & 45 \\
CuO & $1.1 \times 10^{-3}$ & 46 \\
Cu/RGO & $2.8 \times 10^{-3}$ & 47 \\
Cu/CCMs & $1.85 \times 10^{-3}$ & 48 \\
Cu/porous silicon & $1.67 \times 10^{-2}$ & 49 \\
Cu@SBA-15 & $1.73 \times 10^{-2}$ & 50 \\
Cu-DENs & $1.59-2.43 \times 10^{-2}$ & 51 \\
P(AMPS)-Cu(0) hydrogel & $1.72 \times 10^{-3}$ & 52 \\
Cu-P(NIOAM-co-AAc) & $1.73-9.0 \times 10^{-3}$ & 53 \\
Cu-CDs & $1.23(1.26) \times 10^{-2}$ & This work
\end{tabular}

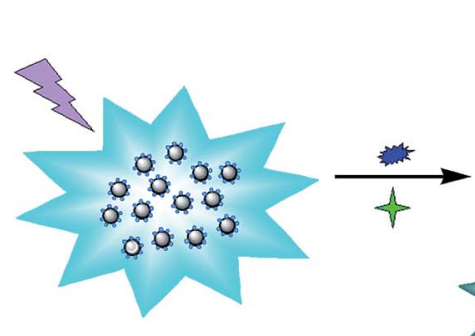

light blue

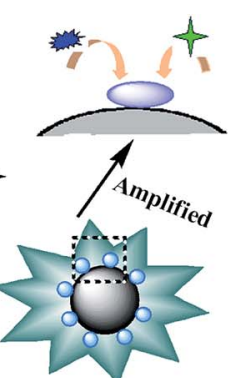

dark blue

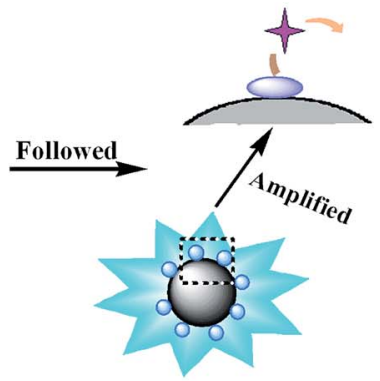

light blue

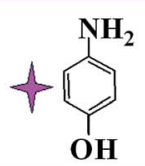

Scheme 1 Schematic layout of reduction reaction mechanism and processing indication of $p$-nitrophenol to $p$-aminophenol catalyzed by the fabricated Cu-doped CDs. 

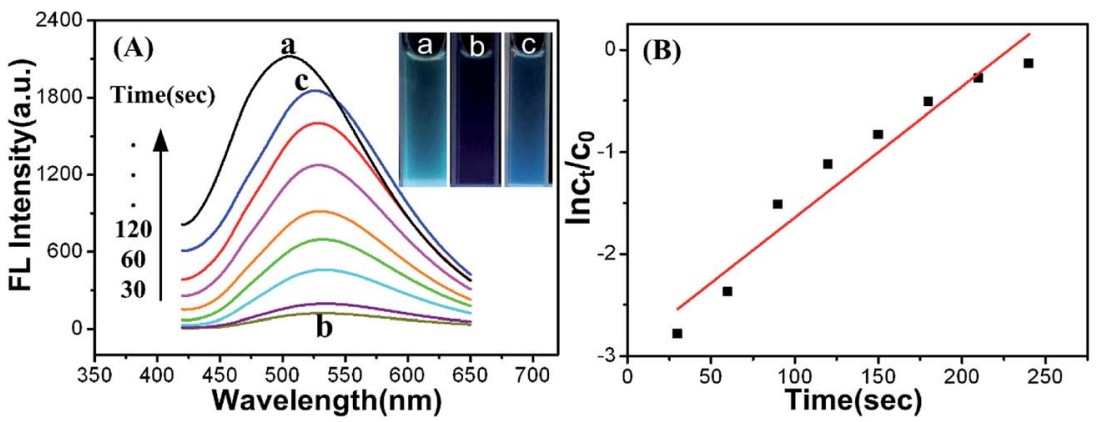

Fig. 6 (A) Time-dependent fluorescence spectra and photographs of $\mathrm{Cu}$-doped $\mathrm{CDs}$ in the presence of $p$-nitrophenol and $\mathrm{NaBH}_{4}$ reaction solution: (a) $0 \mathrm{~s}$, (b) $30 \mathrm{~s}$, (c) $240 \mathrm{~s}$. The fluorescence spectrum was measured with the excitation at $380 \mathrm{~nm}$. (B) Linear relationship of $\ln \left(c_{t} / c_{0}\right)$ as a function of time for the catalytic reduction of $p$-nitrophenol at room temperature.

the doped metal for the researches may be that (i) copper is very cheap and abundantly available in the crust of Earth and, (ii) $\mathrm{Cu}$ owns good electron accepting and donating abilities, which are beneficial for its application in catalytic reaction. As seen in the Table, our result is comparable with and even superior to other Cu-based catalysts. Due to lack of impetus of electron transfer, it is difficult for bare $\mathrm{CD}$ to absorb $p$-nitrophenol onto the catalysts, resulting in inactivity toward reduction of $p$-nitrophenol.

In the meantime, we measured the fluorescence behavior of $\mathrm{Cu}$-doped $\mathrm{CDs}$ in above catalytic reduction reaction from $p$ nitrophenol to $p$-aminophenol in the presence of $\mathrm{NaBH}_{4}$ (Fig. 6). When the as-prepared Cu-doped CDs were added into the mixture of $p$-nitrophenol and $\mathrm{NaBH}_{4}$, in the beginning, the fluorescence of $\mathrm{Cu}$-doped CDs was weakened dramatically, which was accompanied by dark blue color of the reaction mixture (inset of Fig. 6A). After the reaction system reached stable in 30 seconds, the fluorescence of $\mathrm{Cu}$-doped $\mathrm{CDs}$ was gradually enhanced with the reappearance of light blue color (inset of Fig. 6A), corresponding to the decrease of absorption peak at $400 \mathrm{~nm}$ and the increase of new absorption peak at $c a$. $300 \mathrm{~nm}$ in UV-vis absorption spectra (Fig. 5A), which is the generation of $p$-aminophenol accompanying with the consumption of $p$-nitrophenol. The recovery of fluorescence signal could give an indicator for quantitative monitoring of $p$ nitrophenol. In order to comprehend the mechanism of fluorescence changes of the $\mathrm{Cu}$-doped $\mathrm{CDs}$, the excitation spectra of $\mathrm{Cu}$-doped $\mathrm{CDs}$ were found to overlap with the absorption spectra of $p$-nitrophenol in the presence of $\mathrm{NaBH}_{4}$ (Fig. S8 $\dagger$ ). The IFE is due to the absorption of the excitation or emission light by absorbers in the detection system when the absorption spectra of the absorbers overlaps with the fluorescence excitation or emission spectra of fluorophores. ${ }^{54,55}$ During the reduction of $p$-nitrophenol, its concentration decreases gradually accompanied by the decline of absorbance, resulting in the IFE weakening, thus, the fluorescence of $\mathrm{Cu}$-doped $\mathrm{CDs}$ was recovered gradually. To further understand the effect of $p$ nitrophenol concentration on the fluorescence intensity of $\mathrm{Cu}-$ doped CDs, various concentrations of $p$-nitrophenol from 1.0 $\times 10^{-4}$ to $5.0 \times 10^{-4} \mathrm{~mol} \mathrm{~L}^{-1}$ were added into Tris-HCl buffer solution (0.1 M, pH 8.0) containing the Cu-doped CDs. The fluorescence spectra of the Cu-doped CDs were recorded after adding different amount of $p$-nitrophenol. As expected, the fluorescence intensity was decreased with the concentration of $p$-nitrophenol increasing, which corresponds to enhance of absorbance at $400 \mathrm{~nm}$, as depicted in Fig. S9. $\dagger$ Also, the fluorescence of Cu-doped CDs was recovered with the decrease of absorption at $400 \mathrm{~nm}$ in the process of catalytic reaction, in agreement with the above phenomenon. Therefore, the fluorescence quenching or recovery of the $\mathrm{Cu}$-doped $\mathrm{CDs}$ due to $p$ nitrophenol can be interpreted by the IFE of fluorescence.

Moreover, the rate constants of the above catalytic reactions were calculated from the fluorescence spectra of the Cu-doped CDs catalysts. The plot of $\ln \left(c_{t} / c_{0}\right)$ versus time $t$ was shown in Fig. 6B (the $c_{t} / c_{0}$ was provided from the relative fluorescence intensity $\left(I_{t} / I_{0}\right)$ of Cu-doped CDs at maximum emission). From the linear relationship of $\ln \left(c_{t} / c_{0}\right)$ versus time $(t)$, the values of the rate constants of $p$-nitrophenol reduction is $1.26 \times 10^{-2} \mathrm{~s}^{-1}$, which approximately agrees with the values calculated from relative intensity of absorbance. All of the results suggest that catalytic process can be monitored by the fluorescence behavior of luminescent catalyst itself. Therefore, it undoubtedly provides convenience for a class of reaction without specific spectrophotometric characteristics of the substrates.

\section{Conclusion}

In conclusion, the Cu-doped CDs have been successfully synthesized by a one-step hydrothermal carbonization using $\mathrm{CuCl}_{2}-$ $\cdot 2 \mathrm{H}_{2} \mathrm{O}$ and EDTA as precursors. The catalytic reduction of $p$ nitrophenol in the presence of $\mathrm{NaBH}_{4}$ was chosen as a model reaction to investigate the catalytic activity and fluorescence indication performance of the obtained Cu-doped CDs. The synergistic effect of $\mathrm{Cu}$ and graphitic carbon can result in highly catalytic performance and IFE leads to fluorescence change of the Cu-doped CDs catalyst in the process of catalytic reduction reaction. The values of the rate constants for the hydrogenation reduction of $p$-nitrophenol to $p$-aminophenol calculated from the absorbance change of $p$-nitrophenol are consistent with that from the fluorescence intensity change of Cu-doped CDs. Our results indicate that the process of catalytic reduction reaction of $p$ nitrophenol can be monitored by the fluorescence property of catalyst itself. These unique properties would provide an ideal platform to monitor the catalytic process with IFE of fluorescence. 


\section{Acknowledgements}

This work was financially supported by the National Natural Science Foundation of China (No. 21175003 and 21375003).

\section{References}

1 K. M. Choi, K. Na, G. A. Somorjai and O. M. Yaghi, J. Am. Chem. Soc., 2015, 137, 7810-7816.

2 J. Deng, P. Ren, D. Deng and X. Bao, Angew. Chem., Int. Ed., 2015, 54, 2100-2104.

3 D. Jampaiah, T. S. Reddy, A. E. Kandjani, P. Selvakannan, Y. M. Sabri, V. E. Coyle, R. Shukla and S. K. Bhargava, J. Mater. Chem. B, 2016, 4, 3874-3885.

4 W. Zhang, Z. Zhou, X. Shan, R. Xu, Q. Chen, G. He, X. Sun and H. Chen, New J. Chem., 2016, 40, 4769-4774.

5 X. Guo, Y. Wang, F. Wu, Y. Ni and S. Kokot, Analyst, 2015, 140, 1119-1126.

6 J. Mu, Y. He and Y. Wang, Talanta, 2016, 148, 22-28.

7 S. Xu, J. Li, X. Li, M. Su, Z. Shi, Y. Zeng and S. Ni, Microchim. Acta, 2016, 183, 667-673.

8 X. Yi, W. Dong, X. Zhang, J. Xie and Y. Huang, Anal. Bioanal. Chem., 2016, 1-8.

9 M. E. Amato, G. Ansanelli, S. Fisichella, R. Lamanna, G. Scarlata, A. P. Sobolev and A. Segre, J. Agric. Food Chem., 2004, 52, 823-831.

10 K. Tanaka, N. Kitamura, K. Naka and Y. Chujo, Chem. Commun., 2008, 6176-6178.

11 R. Purbia and S. Paria, Biosens. Bioelectron., 2016, 79, 467475.

12 L.-M. Shen, Q. Chen, Z.-Y. Sun, X.-W. Chen and J.-H. Wang, Anal. Chem., 2014, 86, 5002-5008.

13 H. Li, W. Kong, J. Liu, N. Liu, H. Huang, Y. Liu and Z. Kang, Carbon, 2015, 91, 66-75.

14 H. Tao, K. Yang, Z. Ma, J. Wan, Y. Zhang, Z. Kang and Z. Liu, Small, 2012, 8, 281-290.

15 R. C. Green, L. S. Schneider, D. A. Amato, A. P. Beelen, G. Wilcock, E. A. Swabb, K. H. Zavitz and T. P. S. Group, J. Am. Med. Assoc., 2009, 302, 2557-2564.

16 C.-W. Lai, Y.-H. Hsiao, Y.-K. Peng and P.-T. Chou, J. Mater. Chem., 2012, 22, 14403-14409.

17 Y. Li, Y. Zhao, H. Cheng, Y. Hu, G. Shi, L. Dai and L. Qu, J. Am. Chem. Soc., 2011, 134, 15-18.

18 W. Shi, Q. Wang, Y. Long, Z. Cheng, S. Chen, H. Zheng and Y. Huang, Chem. Commun., 2011, 47, 6695-6697.

19 Z. Ma, H. Ming, H. Huang, Y. Liu and Z. Kang, New J. Chem., 2012, 36, 861-864.

20 W. Yang, T. Huang, M. Zhao, F. Luo, W. Weng, Q. Wei, Z. Lin and G. Chen, Talanta, 2017, 164, 1-6.

21 B. C. M. Martindale, G. A. M. Hutton, C. A. Caputo and E. Reisner, J. Am. Chem. Soc., 2015, 137, 6018-6025.

22 A. B. Bourlinos, A. Bakandritsos, A. Kouloumpis, D. Gournis, M. Krysmann, E. P. Giannelis, K. Polakova, K. Safarova, K. Hola and R. Zboril, J. Mater. Chem., 2012, 22, 2332723330.

23 Q. Xu, Y. Liu, R. Su, L. Cai, B. Li, Y. Zhang, L. Zhang, Y. Wang, Y. Wang, N. Li, X. Gong, Z. Gu, Y. Chen, Y. Tan,
C. Dong and T. S. Sreeprasad, Nanoscale, 2016, 8, 1791917927.

24 W. Wu, L. Zhan, W. Fan, J. Song, X. Li, Z. Li, R. Wang, J. Zhang, J. Zheng, M. Wu and H. Zeng, Angew. Chem., 2015, 127, 6640-6644.

25 H. Li, Z. Kang, Y. Liu and S.-T. Lee, J. Mater. Chem., 2012, 22, 24230-24253.

26 S. N. Baker and G. A. Baker, Angew. Chem., Int. Ed., 2010, 49, 6726-6744.

27 W. Lu, X. Qin, S. Liu, G. Chang, Y. Zhang, Y. Luo, A. M. Asiri, A. O. Al-Youbi and X. Sun, Anal. Chem., 2012, 84, 5351-5357.

28 J. Zhou, Z. Sheng, H. Han, M. Zou and C. Li, Mater. Lett., 2012, 66, 222-224.

29 B. De and N. Karak, RSC Adv., 2013, 3, 8286-8290.

30 S.-L. Hu, K.-Y. Niu, J. Sun, J. Yang, N.-Q. Zhao and X.-W. Du, J. Mater. Chem., 2009, 19, 484-488.

31 T. Shao, P. Zhang, L. Tang, S. Zhuo and C. Zhu, Microchim. Acta, 2015, 182, 1431-1437.

32 J. Lu, J.-x. Yang, J. Wang, A. Lim, S. Wang and K. P. Loh, ACS Nano, 2009, 3, 2367-2375.

33 J. Xia, S. Yuan, Z. Wang, S. Kirklin, B. Dorney, D.-J. Liu and L. Yu, Macromolecules, 2010, 43, 3325-3330.

34 H. C. Schniepp, J.-L. Li, M. J. McAllister, H. Sai, M. HerreraAlonso, D. H. Adamson, R. K. Prud'homme, R. Car, D. A. Saville and I. A. Aksay, J. Phys. Chem. B, 2006, 110, 8535-8539.

35 P. Gayathri and A. Senthil Kumar, Langmuir, 2014, 30, 10513-10521.

36 M. Röckert, M. Franke, Q. Tariq, S. Ditze, M. Stark, P. Uffinger, D. Wechsler, U. Singh, J. Xiao and H. Marbach, Chem.-Eur. J., 2014, 20, 8948-8953.

37 Z. Niu, Q. Peng, M. Gong, H. Rong and Y. Li, Angew. Chem., 2011, 123, 6439-6443.

38 J. Li, C.-y. Liu and Y. Liu, J. Mater. Chem., 2012, 22, 84268430.

39 P. Zhang, C. Shao, Z. Zhang, M. Zhang, J. Mu, Z. Guo and Y. Liu, Nanoscale, 2011, 3, 3357-3363.

40 M. Zhu, C. Wang, D. Meng and G. Diao, J. Mater. Chem. A, 2013, 1, 2118-2125.

41 Y.-g. Wu, M. Wen, Q.-s. Wu and H. Fang, J. Phys. Chem. C, 2014, 118, 6307-6313.

42 Z. Jiang, J. Xie, D. Jiang, X. Wei and M. Chen, CrystEngComm, 2013, 15, 560-569.

43 F. Xia, V. Perebeinos, Y.-m. Lin, Y. Wu and P. Avouris, Nat. Nanotechnol., 2011, 6, 179-184.

44 N. Liu, F. Luo, H. Wu, Y. Liu, C. Zhang and J. Chen, Adv. Funct. Mater., 2008, 18, 1518-1525.

45 P. Deka, R. C. Deka and P. Bharali, New J. Chem., 2014, 38, 1789-1793.

46 A. Bhattacharjee and M. Ahmaruzzaman, Mater. Lett., 2015, 161, 79-82.

47 R. Krishna, D. M. Fernandes, J. Ventura, C. Freire and E. Titus, Int. J. Hydrogen Energy, 2016, 41, 11608-11615.

48 X. Cheng, A. Fu, H. Li, Y. Wang, P. Guo, J. Liu, J. Zhang and X. S. Zhao, ACS Sustainable Chem. Eng., 2015, 3, 2414-2422.

49 M. Halim, W. Tan, N. Bakar and M. Bakar, Materials, 2014, 7, 7737. 
50 B. K. Ghosh, S. Hazra, B. Naik and N. N. Ghosh, Powder Technol., 2015, 269, 371-378.

51 M. Nemanashi and R. Meijboom, J. Colloid Interface Sci., 2013, 389, 260-267.

52 N. Sahiner and O. Ozay, Curr. Nanosci., 2012, 8, 367-374.
53 H. F. Zahoor, T. Sakhawat, R. Khan Shanza, F. Kanwal, M. Usman and R. Begum, Materials Science-Poland, 2015, 33, 185-192.

54 G. Gabor and D. R. Walt, Anal. Chem., 1991, 63, 793-796.

55 D. M. Jordan, D. R. Walt and F. P. Milanovich, Anal. Chem., 1987, 59, 437-439. 